\title{
TRITIUM GAS FLOW DYNAMICS THROUGH THE SOURCE AND TRANSPORT SYSTEM \\ OF THE KATRIN EXPERIMENT
}

O.B. Malyshev ${ }^{\mathrm{a}}$, Chr. Day ${ }^{\mathrm{b}}, \mathrm{X} . \mathrm{Luo}^{\mathrm{b}}$ and F. Sharipov ${ }^{\mathrm{c}}$

\footnotetext{
${ }^{a}$ ASTeC, CCLRC Daresbury Laboratory, Warrington, Cheshire, WA4 4AD, United Kingdom

${ }^{\mathrm{b}}$ Forschungszentrum Karlsruhe, Institute for Technical Physics, PO Box 3640, 76021 Karlsruhe, Germany

${ }^{c}$ Departamento de Física, Universidade Federal do Paraná, Caixa Postal 19044, Curitiba 81531900, Brazil
}

Paper is submitted to Journal of Vacuum Science and Technology 


\title{
TRITIUM GAS FLOW DYNAMICS THROUGH THE SOURCE AND \\ TRANSPORT SYSTEM OF THE KATRIN EXPERIMENT
}

\author{
O.B. Malyshev ${ }^{a *}$, Chr. Day $^{\mathrm{b}}$, X. Luo ${ }^{\mathrm{b}}$ and F. Sharipov ${ }^{\mathrm{c}}$ \\ a ASTeC, CCLRC Daresbury Laboratory, Warrington, Cheshire, WA4 4AD, United Kingdom \\ b Forschungszentrum Karlsruhe, Institute for Technical Physics, PO Box 3640, 76021 Karlsruhe, Germany \\ ${ }^{c}$ Departamento de Física, Universidade Federal do Paraná, Caixa Postal 19044, Curitiba 81531-900, Brazil
}

\begin{abstract}
The source and transport system of the Karlsruhe Tritium Neutrino experiment (KATRIN) must provide a significant reduction of tritium flow and gas density. It comprises a $10 \mathrm{~m}$ long windowless source tube, where the tritium gas is injected, followed by a differential pumping system and a cryogenic pumping system. The primary challenge of the analysis is given by the fact that the gas flow changes from viscous flow regime inside the source tube to transitional flow regime at the first pumping stages, and to molecular flow regime at the remaining stages of the differential pumping system and further downstream. A strong molecular beaming effect must be considered. This paper presents the results of calculations of gas density and flow for the complete source and differential pumping system. It is shown that a total flow rate reduction factor of $1.4 \cdot 10^{-8}$ can be attained, which is one of the prerequisites to achieve XHV conditions in the spectrometers used in the downstream end of the experiment.
\end{abstract}

Keywords: Viscous, Transitional and Molecular flow regime; Monte Carlo simulation; Differential pumping; Sticking probability; Capture coefficient; Turbo-molecular pump.

*e-mail: o.b.malyshev@dl.ac.uk 


\section{INTRODUCTION}

The Karlsruhe Tritium Neutrino experiment (KATRIN) is a large vacuum system and aims to measure the mass of the electron antineutrino from the $\beta$-decay of tritium with unprecedented sensitivity. ${ }^{1,2}$ To achieve this purpose, the injected tritium gas flow has to be significantly reduced along the beamline by means of a modular differential pumping system. An international collaboration will construct and operate the experiment in the European Tritium Laboratory on the site of Forschungszentrum Karlsruhe. A detailed description of the KATRIN vacuum system, its requirements and challenges can be found in Ref. [2].

The KATRIN vacuum system has an overall length of about $70 \mathrm{~m}$, comprising four main parts: a gaseous tritium source, a transport section, a system of two electrostatic filters (pre- and main spectrometer) and the electron detector; Figure 1 shows an overall layout of KATRIN. In the centre there is the windowless gaseous tritium source (WGTS) which has to provide a given strength of the decay signal. To achieve that, a cross-section related molecular column density of $N=0.5 \times 10^{22} \mathrm{~m}^{-2}$ at the temperature $T=27 \mathrm{~K}$ must be maintained in the $10 \mathrm{~m}$ long source tube. This is achieved by a continuous tritium inlet gas flow in the middle and by continuous tritium pumping at its ends. The required column density corresponds to a continuous tritium throughput of approximately $1.8 \mathrm{mbar} \cdot 1 / \mathrm{s}$ (referred to the temperature $273.15 \mathrm{~K}$ ) at an injection pressure of about $3 \cdot 10^{-3}$ mbar. This density defines the production rate of the electrons and antineutrinos, which is given by the number of tritium molecules contained in the source tube volume. The WGTS is symmetric and features two identical differential pumping systems (DPS), one at each side. The one in the rear direction (see Figure 2) will reduce the tritium flow to a rear system containing an electron gun used for system calibration. In the other, forward, direction, two differential pumping systems DPS1-F and DPS2-F (see Figure 1 and Figure 2) and a cryogenic pumping system (CPS) are designed to reduce significantly the tritium flow. These pumping systems are surrounded by sophisticated superconducting magnets to transport adiabatically the 
decay electrons ( $ß$-particles) to the spectrometers [3]. To achieve the unprecedented sensitivity of $0.2 \mathrm{eV}$, a pressure below $10^{-11}$ mbar will be required in the electrostatic tandem spectrometer, with a negligible tritium partial pressure (of the order of $10^{-20} \mathrm{mbar}$ ).

\section{FLOW RATE REDUCTION FACTOR REQUIREMENTS}

The prime objective of the KATRIN beamline vacuum pumping system is to provide a significant reduction of the tritium flow rate. On the other hand, the beamline has to be kept as short as possible due to operational constraints; as the beamline is operated under cryogenic conditions and the $\beta$-particles are guided via superconducting magnets, this has major cost implications. In order to come up with an optimised design, one has to develop a model of the gas flow dynamics through the source and transport system, such that the number of pumping stages, the number of pumps, their pumping speed etc can be varied and its influence on the overall flow rate reduction can be studied. This paper therefore presents a complete description of the beamline in terms of flow rates and density.

Based on the symmetry of the WGTS (see Figure 1), it can be assumed that $50 \%$ of the injected gas throughput is directed into the forward direction of the beamline, about $1 \mathrm{mbar} \cdot 1 / \mathrm{s}$. Then there are two downstream limitations to meet:

1. The maximum allowed tritium flow into $\mathrm{CPS}$ is $1 \mathrm{Ci} / 60$ days, due to safety regulation reasons, equal to $8 \cdot 10^{-8} \mathrm{mbar} \cdot 1 / \mathrm{s}$. This leads to the required flow rate reduction factor $\mathrm{K}$ greater than $1.4 \cdot 10^{-8}$ for the complete differential pumping section (DPS1-F\&DPS2-F).

2. It is crucial for the KATRIN experiment that the spectrometers are kept essentially free of tritium. The maximum allowed tritium flow rate into the main spectrometer is defined by the experimental background caused by the decay of tritium molecules in the main spectrometer and shall be maximum $10^{-3}$ counts/s (which gives a residual tritium partial pressure in the main spectrometer of $\mathrm{P}_{\mathrm{MS}}<10^{-20} \mathrm{mbar}$ ). It has been estimated that a tritium flow rate of $\sim 10^{-14} \mathrm{mbar} \cdot 1 / \mathrm{s}$ into the pre-spectrometer will ensure an acceptably 
low background increase in the main spectrometer. ${ }^{2}$ This leads to the required flow rate reduction factor $\mathrm{K}$ from source to spectrometer inlet of approx. $10^{-14}$.

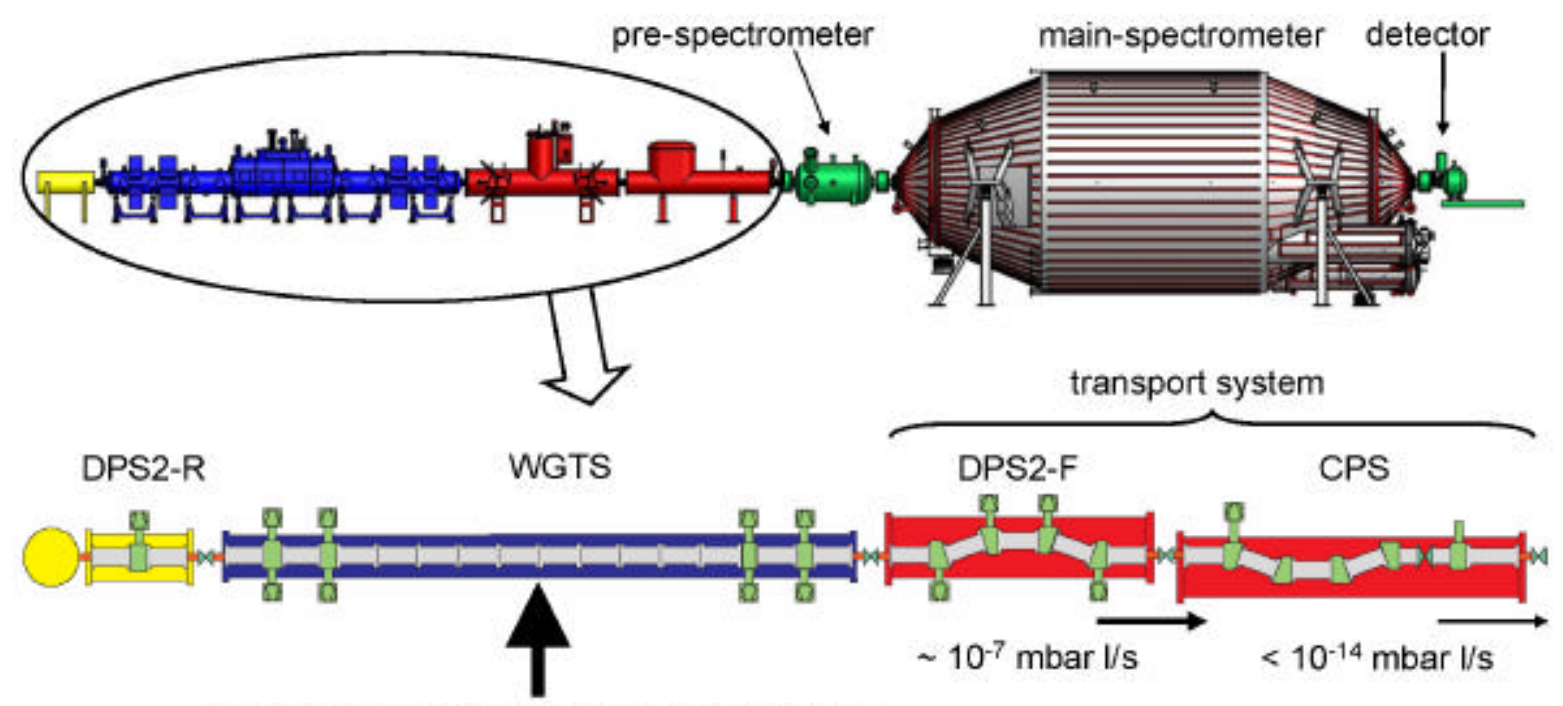

tritium injection: $1.8 \mathrm{mbar} \mathrm{l} / \mathrm{s}=1.7 \cdot 10^{11} \mathrm{~Bq} / \mathrm{s}$

Figure 1. KATRIN layout.
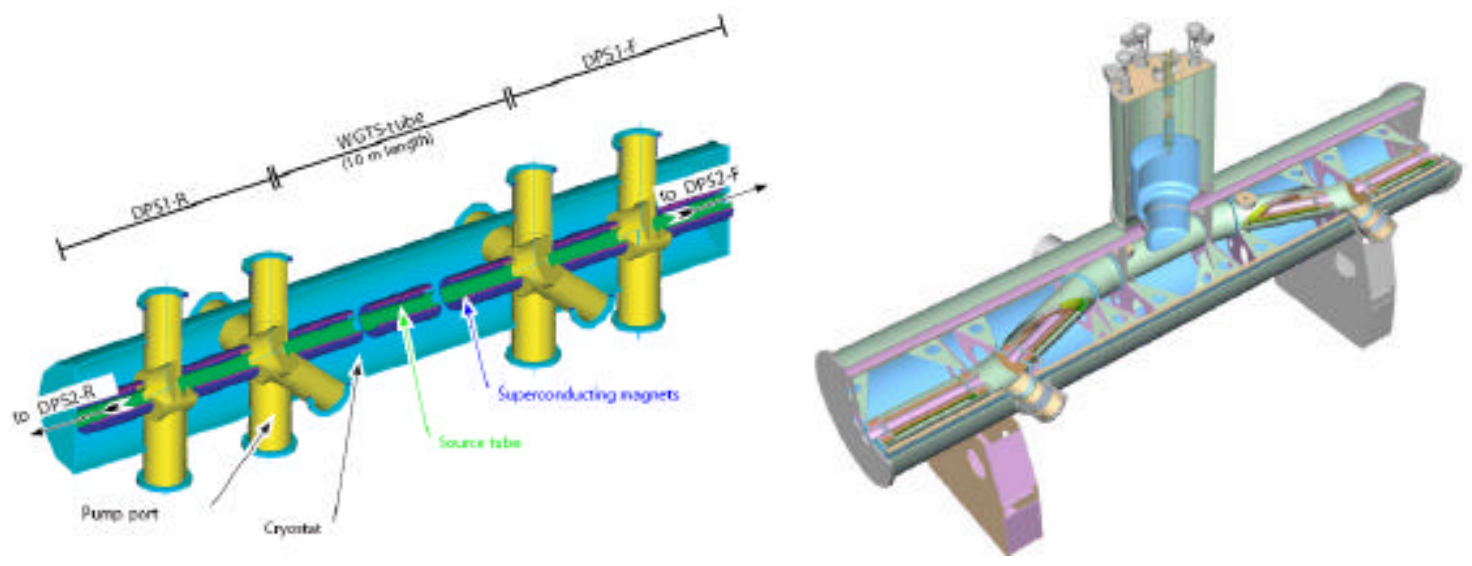

Figure 2. DPS1-F and DPS2-F layout. 


\section{MODELING OF WGTS AND DIFFERENTIAL PUMPING SYSTEM}

The difficulty of the analysis is that the gas flow changes from viscous flow regime inside WGTS to transitional flow regime at the first pumping stage, and finally to molecular flow regime throughout the remaining part of the transport system. The flow regime of rarefied gases is described by the Knudsen number $\mathrm{Kn}$, which is defined as the ratio of the molecular mean free path and the tube radius, or the rarefaction parameter $\delta$, which is proportional to the inverse Knudsen number. The transitional regime is characterized by values of $\mathrm{Kn} \sim 1$, or $\delta \sim 1$ respectively. At the injection point of the WGTS source tube (tritium, $27 \mathrm{~K}, \mathrm{~d}=90 \mathrm{~mm}$ ) a rarefaction parameter is $\delta=20$, which clearly indicates viscous regime. At the exit point the rarefaction parameter is small $(\delta<<1)$, i.e. the gas flow is free molecular.

For simple geometries like the source tube, the kinetic Boltzmann equation can be solved for the whole range of gas rarefaction. However, outside of the tube the geometry of the system is very complicated so that another method must be used. One of the most often chosen methods is the diffusion model. But preliminary analyses have shown that in the case of the molecular gas flow regime there is a strong molecular beaming effect, therefore diffusion $1 \mathrm{D}$ models lead to significant errors and should not be used, and 3D modelling must be applied instead. This is why we are using the test particle Monte Carlo method which is quite appropriate for free molecular flows through complicated geometrical systems.

Another problem comes with the use of numerical methods: the calculations should be completed within reasonable time, but the calculation time increases with the number of test particles, the complexity of the model, and the ratio between largest and smallest size of the model. In addition, for the KATRIN transport system, characterized by a required flow rate reduction factor in the order of $\mathrm{K} \approx 10^{-14}$, one needs to generate at least a factor 100 more molecules to obtain the modelling result with reasonable good statistics. This will require unacceptably long computing time. 
To overcome these difficulties the transport system was analysed firstly element by element. For that purpose, the KATRIN gas flow model was divided according to the real KATRIN hardware sections: WGTS tube, DPS1-F, DPS2-F and CPS (see Figure 1and Figure 2). In a second step additional analysis was performed to merge the individual results.

\subsection{WGTS source tube}

The WGTS source is a tube having a length $L=10 \mathrm{~m}$ and radius $R=45 \mathrm{~mm}$. Tritium is injected in the middle cross section of the tube through many small holes and then it flows to the tube ends where it is pumped by the vacuum systems. The main difficulty of this calculation is that the regime of flow is hydrodynamic in the injection point and it is transitional or practically free molecular at the source ends. Under such condition the problem can be solved only on the basis of the kinetic Boltzmann equation.

To characterize the gas rarefaction, the following parameter $\delta$ is introduced,

$$
\delta=\frac{P R}{\mu v_{m}}, v_{m}=\left(\frac{2 R_{g} T}{m}\right)^{1 / 2}
$$

where $P$ is the pressure, $\mu$ is the gaseous shear viscosity, $v_{m}$ is the most probable molecular speed, $R_{g}$ is the gaseous constant, and $m$ is the molecular mass. The throughput $q$ is expressed via the reduced flow rate $G_{P}$ as

$$
q=\frac{R_{g} T_{0}}{m} \frac{\pi R^{3}}{v_{m}} G_{P}(\delta) \frac{d P}{d x}
$$

where $T_{0}=273.15 \mathrm{~K}$ and $x$ is the longitudinal coordinate with the origin at the middle tube section. The reduced flow rate $G_{P}$ is the function of the local rarefaction parameter $\delta$. The technique of calculation of $G_{P}$ based on the kinetic equation is described in details in Refs. $[4,5,6]$, where the tube length is assumed to be significantly larger than its radius. In the problem 
in question the length-to-radius ratio $L / R$ is about 200 , i.e. the above mentioned assumption is fulfilled.

Once the function $G_{P}(\delta)$ is known Equation (2) can be integrated along the tube from the middle section $(x=0)$ to the tube end $(x=L / 2)$. As a result we obtain:

$$
q=\frac{R_{g} T_{0}}{m} \frac{\pi R^{3}}{v_{m}} G\left(\delta_{e x}, \delta_{i n}\right) \frac{P_{i n}-P_{e x}}{L / 2}
$$

where the quantity $G\left(\delta_{e x}, \delta_{i n}\right)$ is determined by the rarefaction parameter at the source exit $\delta_{e x}$ and by that at the injection point $\delta_{i n}$. It is calculated via the flow rate $G_{P}$ as

$$
G=\frac{1}{\delta_{i n}-\delta_{e x}} \int_{\delta_{e x}}^{\delta_{i n}} G_{P}(\delta) d \delta
$$

To obtain Equations (3) and (4) the relation (1) of the rarefaction parameter to the pressure has been used. In Ref. [4] it was shown that the relation

$$
G\left(\delta_{e x}, \delta_{i n}\right)=G_{P}\left(\frac{\delta_{e x}+\delta_{i n}}{2}\right)
$$

approximates very well the exact integration (4).

Our principal aim is to calculate the throughput $q$ and the injection pressure $P_{\text {in }}$ for a given column density $\mathrm{N}$ of the WGTS source tube, defined as

$$
N=\int_{-L / 2}^{L / 2} n(x) d x
$$

where $n(x)$ is the local number density of tritium, which can be calculated from Eq. (2). If one integrates this equation from the middle section $(x=0)$ to an arbitrary cross section with the coordinate $x$, one obtains

$$
q=R_{g} T_{0} \pi R^{3} v_{m} \frac{\left(n_{i n}-n\right)}{2 x} G_{P}\left(\frac{\delta_{i n}+\delta}{2}\right)
$$


Combining this equation with Eq. (3) we obtain

$$
\frac{2 x}{L}=\frac{n_{i n}-n}{n_{i n}-n_{e x}} \frac{G_{P}\left(\frac{\delta_{i n}+\delta}{2}\right)}{G_{P}\left(\frac{\delta_{i n}+\delta_{e x}}{2}\right)}
$$

So, in practice one calculates the function $x=x(n)$. Then this function is inverted and the column density is calculated by Eq. (6). Thus, it is necessary to fit $P_{i n}$ so that the column density would be equal to its required value, i.e. $N=0.5 \times 10^{22} \mathrm{~m}^{-2}$. Then, the throughput is calculated from Eq. (3).

To calculate the rarefaction parameter $\delta$ some experimental data on the viscosity of tritium are necessary. Unfortunately, no data on the viscosity at a low temperature are available in the open literature. Some data on the viscosity of hydrogen and deuterium at a low temperature are reported in Refs. [7,8,9]. So, the viscosity of tritium $\mu_{T}$ can be calculated via the viscosity of deuterium $\mu_{D}$ assuming that both molecules have the same cross sections. In this case we obtain $\mu_{T}=\sqrt{3 / 2} \mu_{D}$. This relation works very well for a high temperature when the rotations of both molecules $T_{2}$ and $D_{2}$ are classical. At a low temperature every molecule has its own spectrum of the rotational energy, which affects the cross section. An analysis of the experimental data on hydrogen [7] and deuterium [8] showed that the analogous expression $\mu_{D}=\sqrt{2} \mu_{H}$ provides an overstated value of the viscosity $\mu_{D}$ for $7 \%$. So, it is expected that such a relation provides the slightly overstated values of $\mu_{T}$. The discrepancy should be within $5 \%$. Finally, using the experimental value of $\mu_{D}=2.084 \times 10^{-6} \mathrm{~Pa} \mathrm{~s}$ at $T=30 \mathrm{~K}$ reported in Ref. [8] we obtain the viscosity of tritium as $\mu_{T}=0.95 \sqrt{3 / 2} \mu_{D}=2.425 \times 10^{-6} \mathrm{~Pa}$ s. This value was used in our numerical calculations. 
First, the calculations were carried out for the gas flow into vacuum, i.e. when $\delta_{e x}=0$. The density distribution in this case is shown on Figure 3. However, in practice the exit pressure $P_{e x}$ is not so low to assume $\delta_{e x}=0$. Thus, additional calculations were carried out to study the influence of $P_{e x}$ on the column density and throughput. The results of these calculations are presented in Table 1, which show that the influence is significant. In the future, when the pressure $P_{e x}$ is measured other calculations will be carried out for the measured values.

Table 1. Table A: Column density $N$ and throughput $q$ vs exit pressure $P_{e x}$ at $P_{i n}=3.006$ bar.

\begin{tabular}{|c|c|c|}
\hline$P_{e x} / P_{i n}$ & $N / 10^{22}$ & $q$ \\
\hline 0 & 0.5 & 1.853 \\
\hline 0.005 & 0.5007 & 1.851 \\
\hline 0.01 & 0.5014 & 1.848 \\
\hline 0.05 & 0.5007 & 1.824 \\
\hline 0.1 & 0.5167 & 1.788 \\
\hline
\end{tabular}

The present calculations were carried out assuming the complete accommodation of tritium on the tube wall. No data can be found about the accommodation coefficient of this gas at a low temperature. In the work [14] the accommodation coefficient of hydrogen on a glass surface at the room temperature was calculated from the experimental data on the slip coefficient. The obtained value is 0.952 . It is difficult to say if tritium has a lower or higher accommodation coefficient at the low temperature, i.e. at $T=27 \mathrm{~K}$. It just can be said that it can vary in the range from 0.8 to 1 . On the other hand, its influence on the flow rate is significant only in the free molecular regime. To evaluate the influence of the accommodation coefficient on the column density $N$ some additional calculations were carried out for its value equal to 0.8 , which showed 
that the uncertainty of the accommodation coefficient of $20 \%$ causes the uncertainty of the column density $N$ within $1.6 \%$. Thus, the calculations based on the diffuse gas-surface interaction are quite reliable.

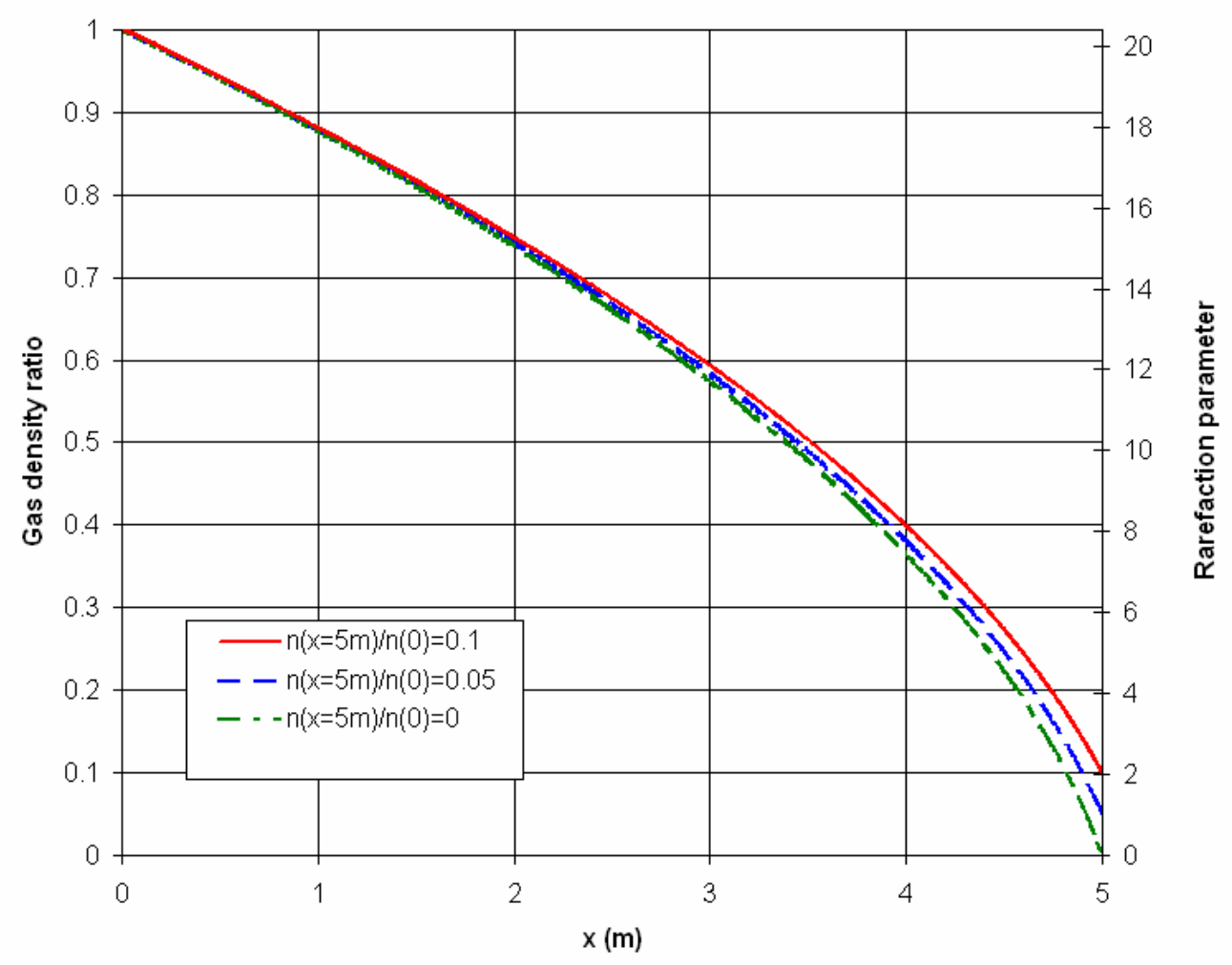

Figure 3. Tritium gas density ratio along WGTS source tube.

\subsection{Modelling of DPS2-F}

In the assessment of the KATRIN vacuum pumping system, the model of DPS2-F was the one to start with, as it was clear that the gas flow regime is molecular and the available Test Particle Monte-Carlo (TPMC) codes can be used without worry about intermolecular collisions. The detailed description of the model, assumptions and the analysis of results of the modelling were published in Ref. [10]. It has been shown that by using the candidate turbo-molecular pump (TMP), characterised by an effective capture probability at the pumping port of $\alpha_{T M P}=0.3$, a 
tritium flow rate reduction factor of the complete DPS2-F system of about $K_{I I}=1 /\left(1.3 \cdot 10^{5}\right)=7.7 \cdot 10^{-6}$ can be achieved. Under these conditions, the calculated gas density ratio over the complete DPS2-F system was $R_{I I}=1 /\left(6.6 \cdot 10^{6}\right)=1.5 \cdot 10^{-7}$.

It is necessary to mention that an accident case was also modelled: if one out of four TMPs connected to DPS2-F is failed, the tritium flow rate reduction factor $K_{I I}{ }^{*}$ of the complete DPS2-F system will be about 30 times larger: $K_{I I}{ }^{*}=2.3 \cdot 10^{-5}$.

\subsection{Modelling of DPS1-F}

From the modelling aspect, DPS1-F is the most challenging part of the transport section. There are transitional flow conditions at the inlet and free molecular flow conditions in the outlet. Unfortunately, there exist no solutions of the kinetic equation for such a complex geometry of the pumping ports.

To have an accurate representation of the flow situation, the Direct Simulation Monte Carlo-approach would be most appropriate. However, such a work would be a very major effort and is not reasonable to do within an ongoing design process. This is why it was decided to use the conventional TPMC method, being aware that the final results might be leading to lower reduction factors, because the intermolecular collisions which neglected in TPMC will reduce the molecular beaming effect, so that introduces some additional safety margin in the overall design and does not play any negative role.

Two DPS1 Monte-Carlo models were built based on the ACCEL design. The core model includes the following elements (see Figure 4):

- An inlet surface with sticking probability $\alpha_{1}$ (Ring 1 in Figure 4).

- A part of WGTS (a 0.9-m long tube with a diameter of $90 \mathrm{~mm}$ which begins at $\mathrm{x}=4 \mathrm{~m}$ in respect to $\mathrm{x}=0$ at the middle of WGTS), the tube ended by a cone ( $\mathrm{x}$ is the axial coordinate). 
- Pumping port 1 with four ducts leading to TMP, four pumping surfaces (Ring 2 to Ring 5) with a capture probability $\alpha_{\text {TMP. }}$ The pumping port is of cubical shape with dimensions of $374 \times 374 \times 250 \mathrm{~mm}^{3}$; the pumping ducts are $656 \mathrm{~mm}$ in length in $250 \mathrm{~mm}$ in diameter.

- A tube between two pumping ports with cones at either side $(1060 \mathrm{~mm}$ long, $90 \mathrm{~mm}$ diameter);

- Pumping port 2 (of the same dimension as above) with two ducts leading to TMP, two pumping surfaces Ring 6 and Ring 7 with a capture probability $\alpha_{\mathrm{TMP}}$; (the pumping ducts are $406 \mathrm{~mm}$ in length and $250 \mathrm{~mm}$ in diameter)

- A tube between the pumping port 2 and the gate valve in front of DPS2 with cones at either side (1060 mm long, $90 \mathrm{~mm}$ diameter).

- An outlet surface Ring 8 with sticking probability $\alpha_{N}$;

- In addition, the Monte Carlo Codes allow setting the few transparent test facets for gas density profiles calculations. The test facets were set along and the main axes and along one pumping duct at each pumping port.

The model contains one inlet surface $(i=1)$, and seven outlet surfaces (the 4 pumps in the first port, the 2 pumps in the second port, and the outlet surface; $i=2 \ldots 8)$. Two different Monte Carlo codes have been used, ${ }^{11,12}$ and no particular deviations have been found.

The Monte-Carlo simulations have been performed the same way as described in [10] when the pumping surfaces (inlet, outlet and pumping surface in pumping ducks) have a sticking probability of 1 and the particles were generated from one of the pumping surfaces, this was repeated four times: i.e. for the inlet, pumping port 1, pumping port 2 and the outlet. Number of generated particles was $10^{7}$ for each run. Results of the modelling were:

- The transmission probability matrix $\mathbf{W}$;

- A number of particles $m_{j}$ passed trough each of 200 elements along the test facets. 


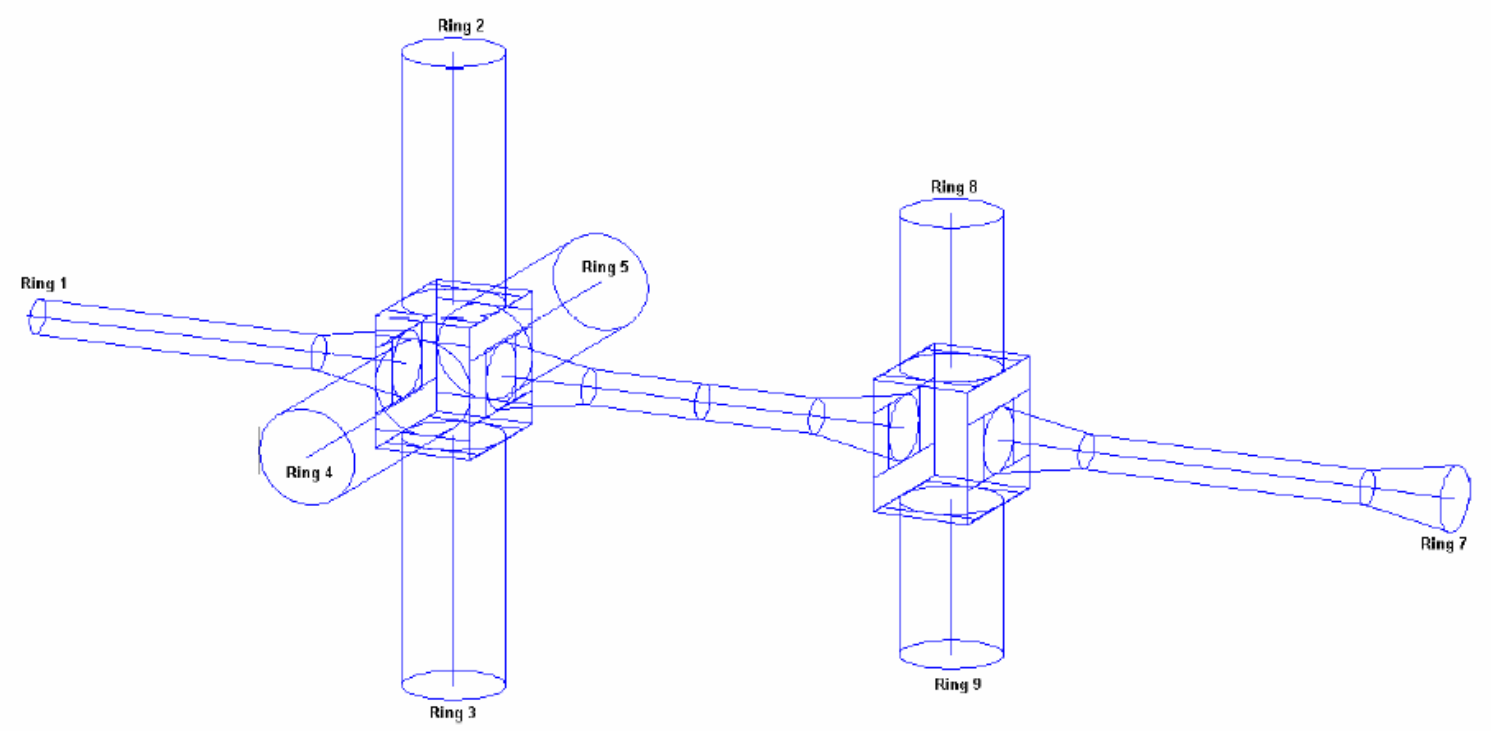

Figure 4. Layout of the core model for DPS1.

\subsubsection{Flow rate reduction factor and gas density ratio as a function of the pump} capture probability

Following the procedure described in [10] a vector $\mathbf{f}$ of the incoming flow $f_{i}$ to each pumping surface can be found by solving the matrix equation with the transmission probability matrix $\mathbf{W}$ for a vector $\mathbf{f}$ :

$$
[\mathbf{E}-\mathbf{W} \cdot \operatorname{diag}(1-\mathbf{a})] \cdot \mathbf{f}=\mathbf{W} \cdot \mathbf{d}
$$

where $\mathbf{E}$ is a unit matrix; $\mathbf{W}$ is the transmission probability matrix; $\operatorname{diag}(1-\alpha)$ is the diagonal matrix of vector (1- $\alpha$ ), where $\alpha$ a vector of capture probability, $\mathbf{d}$ is the gas desorption (or injection) vector. In the case of DPS1, tritium comes from the inlet surface only: $d_{1}>0$ and $d_{i}=0$ for $i=2 \ldots 7$. The sticking probability vector was defined as the following: $\alpha_{1}=0$ (inlet surface), 
$\alpha_{i}=0.3$ for $i=2 \ldots 7$ (TMPs), the capture probability of the outlet surface $\alpha_{N}$ was evaluated with formula (15) from the Monte-Carlo results for DPS2-F [10]: $\alpha_{\mathrm{N} \text { DPS1-F }}=\chi_{\mathrm{DPS} 2-\mathrm{F}}=0.078$,

Having the solutions for $\mathbf{f}$, the gas flows $\mathbf{Q}$ at every boundary surfaces can be calculated from Equation (5).The overall efficiency of DPS1-F is characterized by the flow rate reduction factor $K_{I}$ as the ratio of fluxes at the outlet and inlet of DPS1-F. The efficiency of the differential pumping at the first pumping port with four TMPs and at the second pumping port with two TMPs can be characterized by the individual flow rate reduction factors $K_{I a}$ and $K_{I b}$, i.e. the ratio of fluxes after and before the pumping ports, as following:

$$
K_{I}=\frac{\alpha_{N} f_{N}}{1-\alpha_{1} f_{1}}, \quad K_{I a}=\frac{\sum_{j=6}^{N} \alpha_{i} f_{i}}{1-\alpha_{1} f_{1} \sum_{j=6}^{N} \alpha_{i} f_{i}}, \quad K_{I b}=\frac{\alpha_{N} f_{N}}{\sum_{j=6}^{N} \alpha_{i} f_{i}}
$$

Figure 7 presents the individual pump port flow rate reduction factors together with the overall value $\mathrm{K}_{\mathrm{I}}$. One can see that assuming the pump capture probability of 0.3 , based on the results in [4], the gas flow is reduced $\sim 30$ times after the pumping port 1 and $\sim 10$ times after the pumping port 2, the total flow rate reduction factor is $K_{I} \approx 0.003$.

The gas density ratio $R_{i}$ which is defined as the ratio of the gas density at inlet surface and surface $i$ can be estimated as:

$$
R_{i}=\frac{n_{1}}{n_{i}}=\frac{d_{1}+\left(2-\alpha_{1}\right) f_{1}}{\left(2-\alpha_{i}\right) f_{i}} \frac{A_{i}}{A_{0}}, i=2 \ldots 8
$$

where the $A_{i}$ are the cross sectional areas of corresponding boundary surfaces.

In fact, these two ratios $\left(K_{i}\right.$ and $\left.R_{i}\right)$ do not depend on $\alpha_{1}$, because by changing $\alpha_{1}$ one changes the injected flow $Q_{1}$, but does not change the transmission probabilities between surfaces; therefore the fraction of injected flow pumped by each pump does not depend on the absolute value of this flow (remaining within molecular flow regime). The same considerations 
are valid for the gas density rate. Meanwhile both ratios depend on the sticking probability of outlet surface.

The overall gas density ratio $R_{I}$ (the ratio of the gas density at the DPS1 outlet and intlet) is shown on Figure 6 together with the Gas Flow Rate Reduction Factor. One can see that both the gas density and flow ratios are quite sensitive to the pump capture probability less then $0.2-0.3$, for the higher capture probability this dependence is quite small, that means that the conductance (or transmission probability) of the pumping ducts is a limiting factor for the gas density and flow ratios.

\subsubsection{Gas density ratio along the DPS1}

The MOLFLOW code allows setting a transparent test strips consisting of 100 pieces to count a number of particles passing it through, this can be converted into the gas density profile. In general, this can be done with an approximate formula:

$$
n_{k}=\frac{2 Q m_{k}}{N A_{k} v} \text { for } k=1 \ldots 100
$$

where $n_{k}$ is the gas density near the $k$-th element with surface $A_{k}$ corresponding an injected flux $Q ; v$ is the mean molecular velocity; other parameters are from Monte-Carlo calculations: $N$ is a number of generated test particles and $m_{k}$ is a number of particles passed through the $k$-th element. The approximate nature is coming from uncertainty in the molecular velocity and its non-uniform field. 


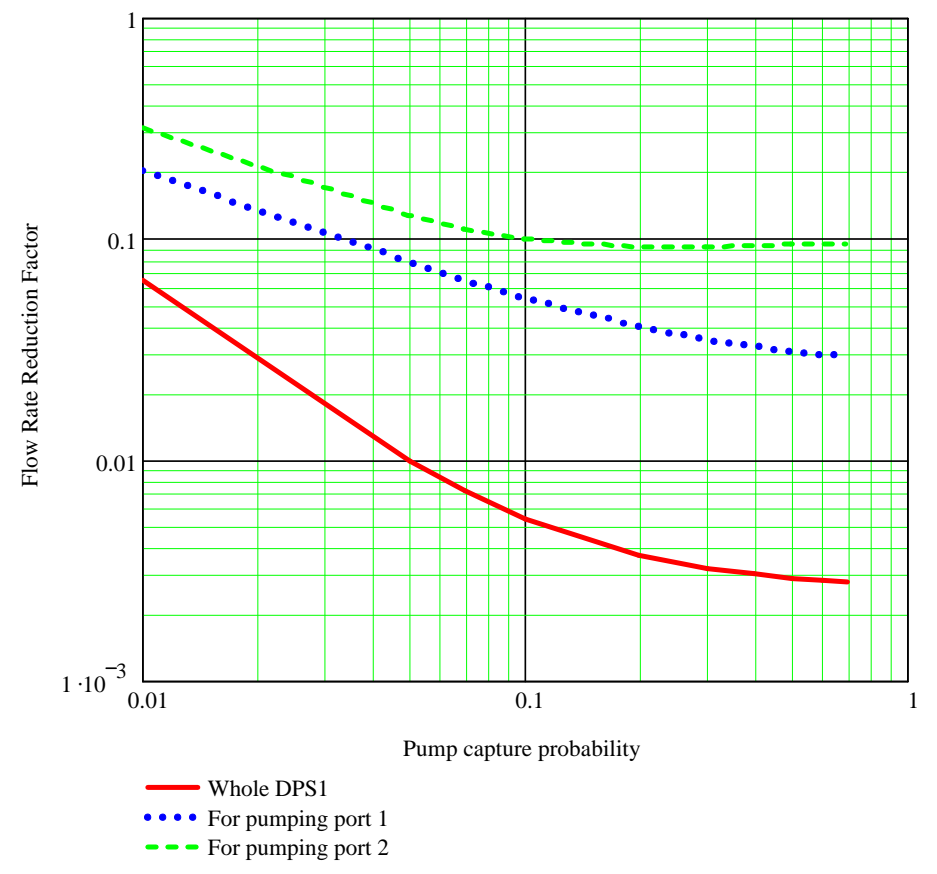

Figure 5. DPS1 Gas Flow Rate Reduction Factor as a function of turbo molecular pump capture probability.

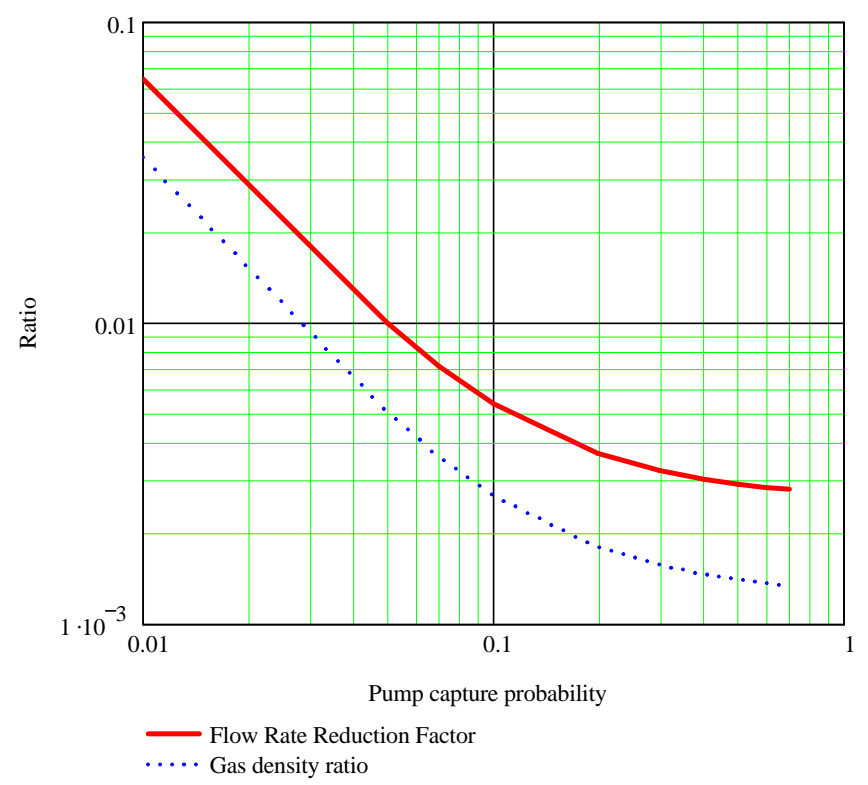

Figure 6. DPS 1 Gas flow rate reduction factor and gas density factor as a function of turbo molecular pump capture probability. 
In the DPS1 model, the molecules which reached the entrance to TMP and were not pumped are included (as they were generated at this surface) in the gas density according to the following expression:

$$
n_{k}=\frac{2 Q}{N v} \sum_{i=0}^{7}\left(f_{i}\left(1-\alpha_{i}\right) \frac{m_{k, i}}{A_{k}}\right) \text { for } k=1 \ldots 100, i=0 \ldots 7
$$

here index $i$ corresponds to the results and parameters for the pumping surface $i, m_{k, i}$ is a number of particles passed through the $k$-th element when desorption in the TPMC modelling were from $i$-th pumping surface.

The test strips were set along the main axis of the DPS1 (2 serial strips: 200 elements). The results of calculations with pump capture probability of 0.3 are shown by a green line on Figure 7. The result are normalised to 1 at $\mathrm{z}=4 \mathrm{~m}$ (the entrance to the model).

The test facets were also set across the vacuum chamber (violet triangles on Figure 7). These facets count particles arriving from one side only. Most of the facets were faces to the inlet and only two facets (with coordinates $\mathrm{z}=5.37 \mathrm{~m}$ and $7.53 \mathrm{~m}$ ) were faces to outlet. At the coordinate $\mathrm{z}=5.37 \mathrm{~m}$ there were two test facets with different orientation; one can clearly see that facets faced to the inlet gives $20 \%$ higher impingement rate than one faced to the outlet. The impingement rate of the facets faced to the inlet is always higher than one for the longitudinal one. This all indicates the molecular beaming effect.

Another interesting effect is that although there is only one source of gas at the inlet the gas density profile shows a pronounced increase after each pumping port which can be explained that some number of particles travel through the pumping port without collisions with its walls and collide with walls of the next tube, after that these particles can diffuse to either end. 


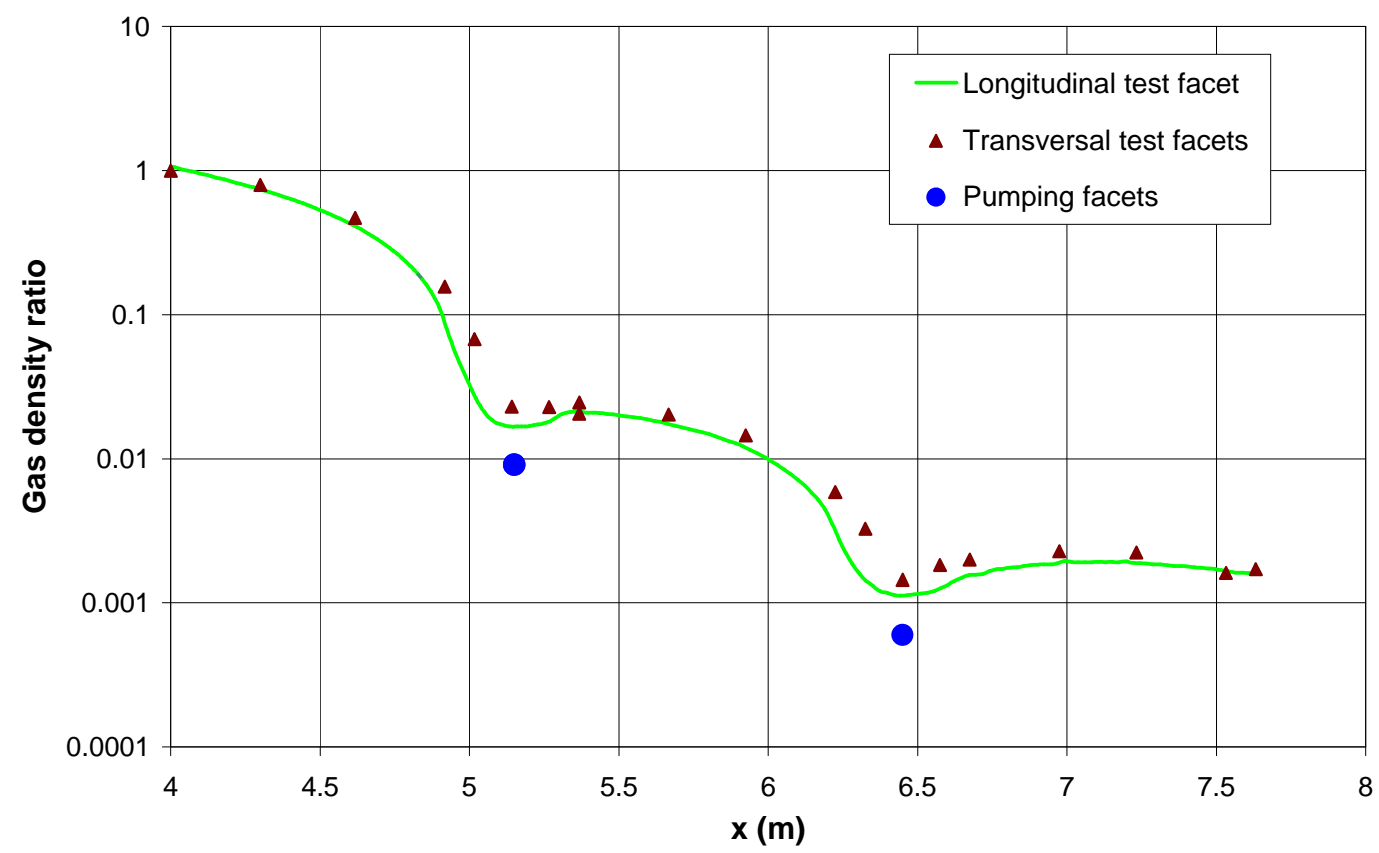

Figure 7. Gas density along DPS1 and at the pumping ports.

\section{GAS FLOW AND DENSITY RATIO OVER ALL TRANPORT LINE}

Consider three serial elements with entrances $a_{1}, a_{2}$ and $a_{3}$ and exits $b_{1}, b_{2}$ and $b_{3}$. When it is necessary to model the flow rate reduction and the gas density rate, the boundary conditions are defined by the following gas flow balance (in dimensionless form, related to the overall inlet gas flow):

$$
Q\left(a_{1}\right)=1 ; \quad Q\left(a_{2}\right)=-Q\left(b_{1}\right) ; \quad Q\left(a_{3}\right)=-Q\left(b_{2}\right) .
$$

For continuity reasons, the condition $Q\left(a_{2}\right)=-Q\left(b_{1}\right)$ is fulfilled when $d_{a 2}=f_{b 1}$ and $d_{b 1}=f_{a 2}$ (see Figure 8), where $f$ is a gas flux to the surface and $d$ is the desorption flux from the surface (in the same terms as used for analysis in [10]). Section 2 can be characterised by its capture probability which is defined as:

$$
\chi_{S e c 2}=1-\frac{f_{a 2}}{d_{a 2}}=1-\frac{f_{a 2}}{f_{b 1}} .
$$


Then the flow $d_{b 1}$ from section 2 to section 1 is equal to:

$$
d_{b 1}=f_{a 2}=\left(1-\chi_{\operatorname{Sec} 2}\right) f_{b 1}
$$

This illustrates that the results for the downstream section 2 are needed to model the upstream Section 1 for obtaining the Section 2 capture probability.

\section{Section $1 \quad$ Section 2}

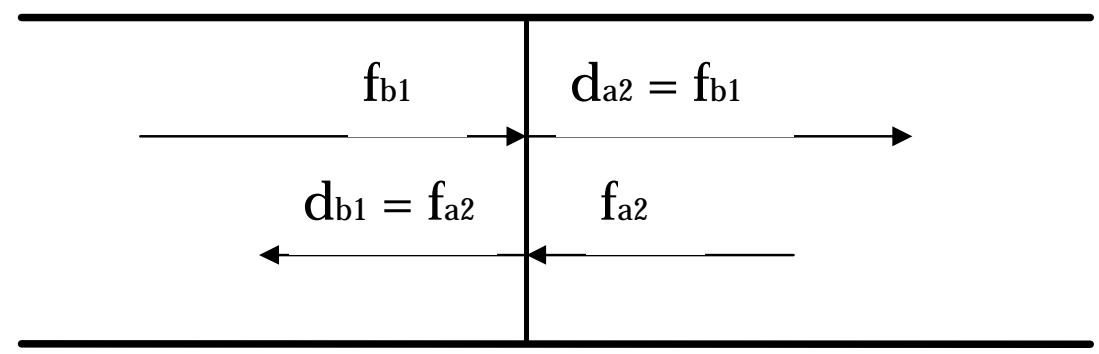

Figure 8. Boundary conditions between two sections.

This method is correct in assumption that the velocity field is the same in either side of the boundary between two sections. In practice, the TPMC code used for this work allows to generate either uniform or cosine low molecular distribution of desorbed molecules which is not the same as for the molecules hitting the boundary between two sections from another side. This introduces the deviation of the results. A separate TPMC model was build by splitting the DPS1-F in two half. Comparing the results of this modelling to one for a fool DPS1-F model has shown that the difference between two models is insignificant (less than $2 \%$ ) when cosine low molecular distribution of desorbed molecules used. 


\subsection{Merging the results for the WGTS source tube and DPS1-F}

It is necessary to mention here that including the 1-m long part of the WGTS source tube in the DPS1 model plays very important role in the model. The molecular velocity distribution formed during passing this 1-m tube builds up a molecular beam which goes directly to the exit without any collisions with walls. The number of these molecules is significant. The model without the 1-m tubular part of the WGTS gives flow ratio reduction factor is about 600 against about 300 in the case with such a tube, i.e. underestimate the molecular beaming effect by factor 2 . If were all WGTS and DPS it the molecular flow regime the model must have been build from the middle of the WFTS and the molecular beaming effect would be even stronger. Meanwhile it should not be forgotten that the gas flow regime there is viscous in the middle and transitional at the end of WGTS, therefore the number of intermolecular collision is growing towards the centre of WGTS, and the beaming effect is demolished.

The conditions to find the correct point of joining of two solutions: WGTS source tube and DPS1 were following:

$$
\begin{gathered}
Q_{W G T S}\left(x_{1}\right)=Q_{D P S 1-F}\left(x_{1}\right) \\
n_{W G T S}\left(x_{1}\right)=n_{D P S 1-F}\left(x_{1}\right) \\
\frac{d n_{W G T S}\left(x_{1}\right)}{d x}=\frac{d n_{D P S 1-F}\left(x_{1}\right)}{d x}
\end{gathered}
$$

In both models: for the WGTS source tube and DPS1, $Q$ is sufficient to find by $n(x)$ and $(d n / d x)$, the conditions (18) and (19) are important to check that there is an overlapping interval where two solutions match each other. If the centre of the WGTS corresponds to $x=0$, then the entrance to the DPS1-F model is at $\mathrm{x}=4 \mathrm{~m}$. It was found that the conditions (17) are fulfilled for the source calculation with $P_{e x} / P_{i n}=0.04$ at the interval $4.7 \mathrm{~m}<x_{1}<5 \mathrm{~m}$ as it is shown on Figure 9. 


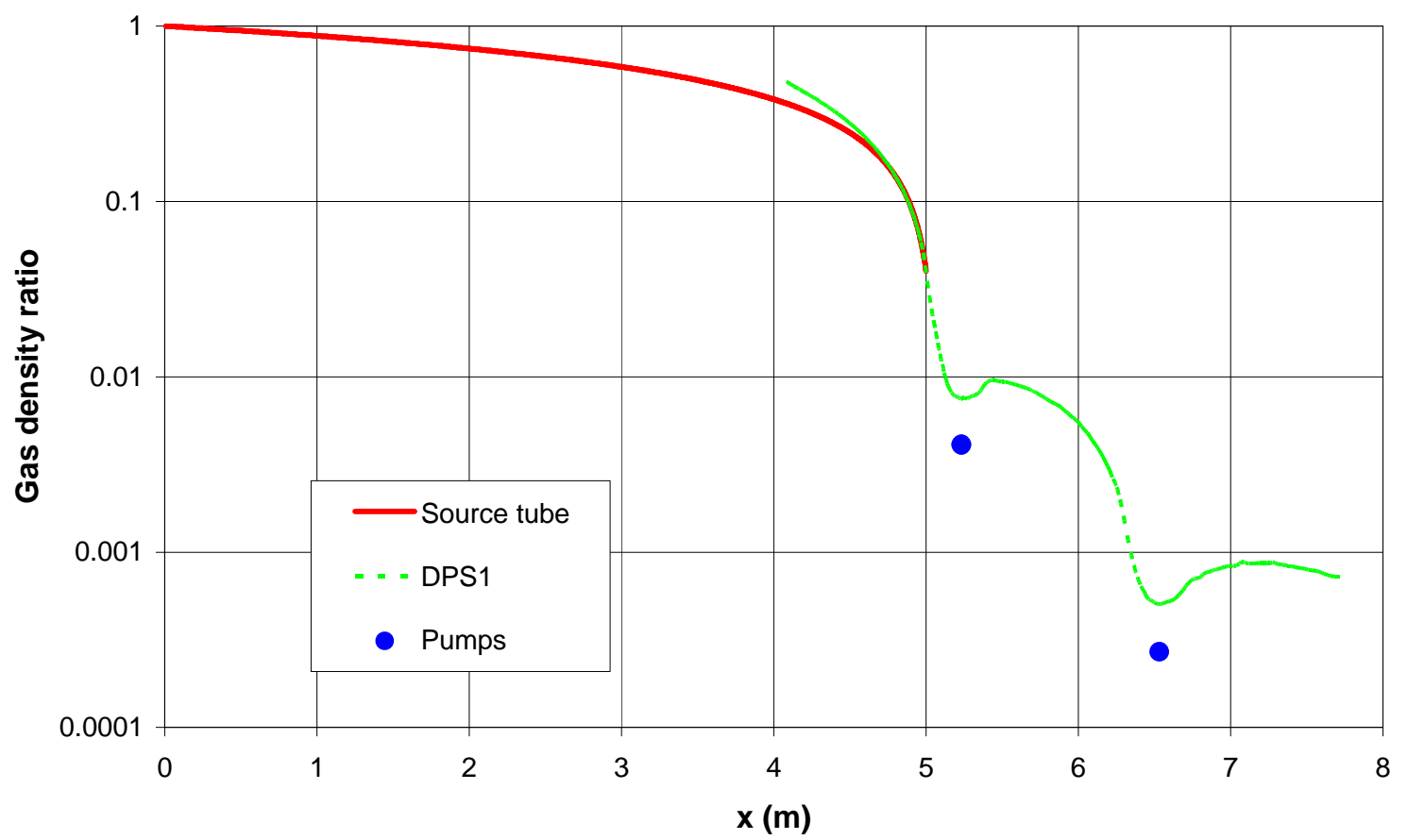

Figure 9. Merging solutions for WGTS source tube and the complete DPS.

\subsection{Merging the results for the DPS1-F and DPS2-F}

The entrance boundary conditions for DPS2-F are:

$$
\begin{aligned}
& Q_{D P S 1-F}(\text { outlet })=Q_{D P S 2-F}(\text { inlet }) \\
& n_{D P S 1-F}(\text { outlet })=n_{D P S 2-F}(\text { inlet })
\end{aligned}
$$

Now it is possible to plot the flux rate and the gas density ratio along the transport line. The result for the flux rate and the gas density rate shown in Figure 10 and Figure 11 for the turbo pump capture probability $\alpha=0.3$ which was estimated in [10]. The overall gas density ratio along WGTS, DPS1-F and DPS2-F is $R=2.0 \cdot 10^{-10}$ and Gas Flow Rate Reduction Factor is $K=1.4 \cdot 10^{-8}$. The most important conclusion from the diagrams above is, that the requirement to have a flow rate reduction factor of $1.4 \cdot 10^{-8}$ can be met. However, we have to have in mind that there is transitional flow at around the first pumping port of DPS1, which means that the flow 
rate reduction factors as calculated with TPMC method may be not very accurate. Moreover, there is the influence of temperature gradients etc., so that we have to conclude that there is no safety margin included in the design of DPS1 and DPS2, with respect to the flow rate reduction. Consequently, the design of the downstream sections of the transport system must account for the additional contingency needed. This will be discussed in detail in the following section.

\section{CONCLUSIONS FOR THE REMAINING DOWNSTREAM TRANSPORT}

\section{SYSTEM}

As it was explained in the beginning of this paper, the required flow rate reduction factor $\mathrm{K}$ from source to spectrometer inlet of approx. $10^{-14}$. The flow rate reduction factor from source to the outlet of DPS2F is $K_{D P S 1+D P S 2}=1.4 \cdot 10^{-8}$. Therefore, the remaining part of the transport system comprising the cryogenic pumping system (CPS) and the prespectrometer (PS) should provide a minimum additional flow rate reduction of about $7 \cdot 10^{-7}$, it should also preferably have some safety margin: $1 / 30$ for an accident with one failed TMP and about factor $1 / 2$ for some differences between the model and a real design; hence there is a need of $K_{C P S+P S} \approx 10^{-8}$.

\section{CONCLUSIONS.}

A numerical model results for the WGTS source tube as well as the TPMC model results for DPS1-F are presented. It was shown that the strong molecular beaming effect limiting the DPS1F Gas Flow Rate Reduction Factor to $K_{I} \approx 3 \cdot 10^{-3}$. These results were analysed together with results published earlier for other part of KATRIN: DPS2-F. The calculated gas density ratio along WGTS source tube, DPS1-F and DPS2-F is $R=2.0 \cdot 10^{-10}$ and Gas Flow Rate Reduction Factor is $K=1.4 \cdot 10^{-8}$. The remaining part of the transport system comprising CPS and PS should provide an additional flow rate reduction $10^{-8}$. 


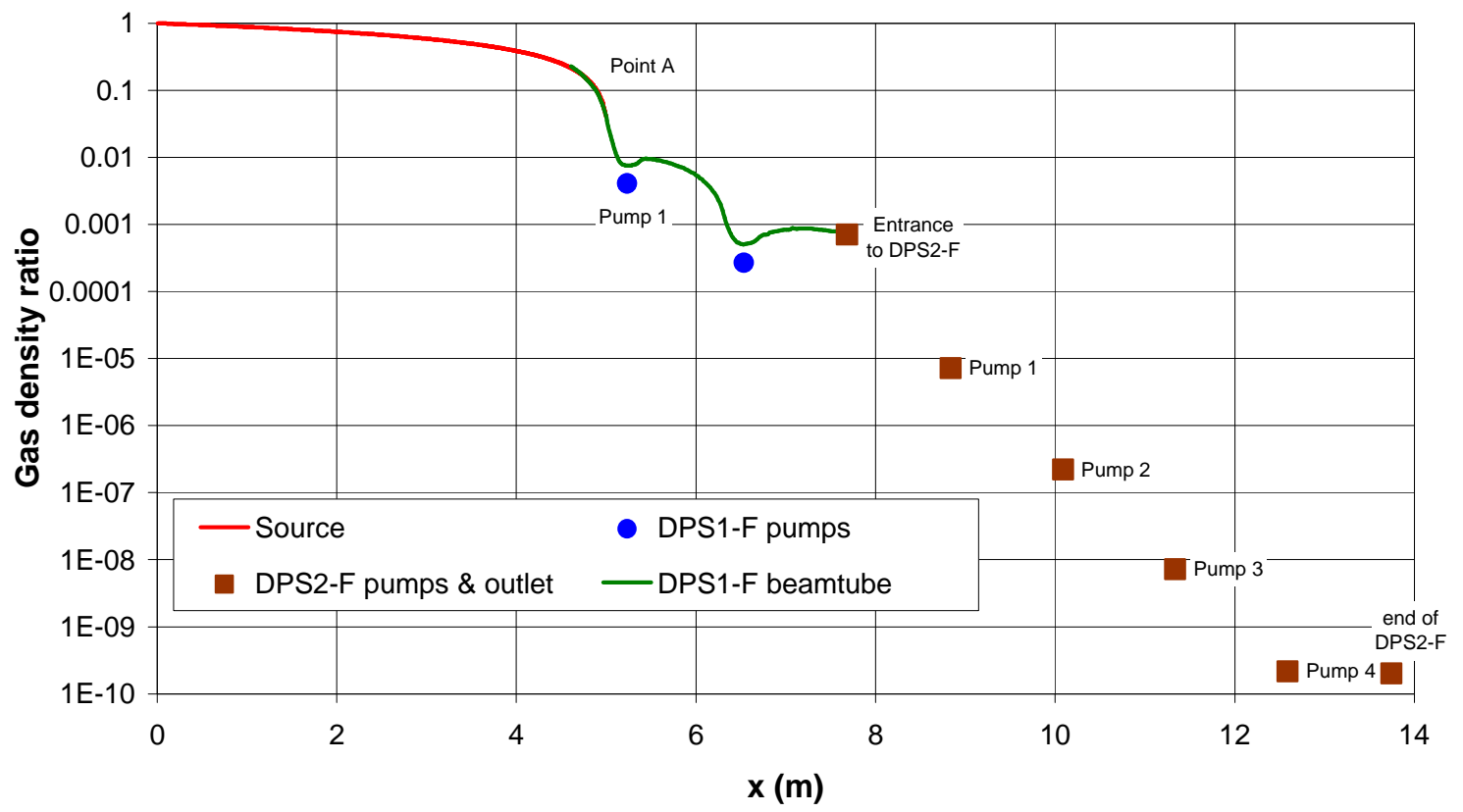

Figure 10. The gas density ratio for the complete differential pumping system (values for DPS2-F from [4].

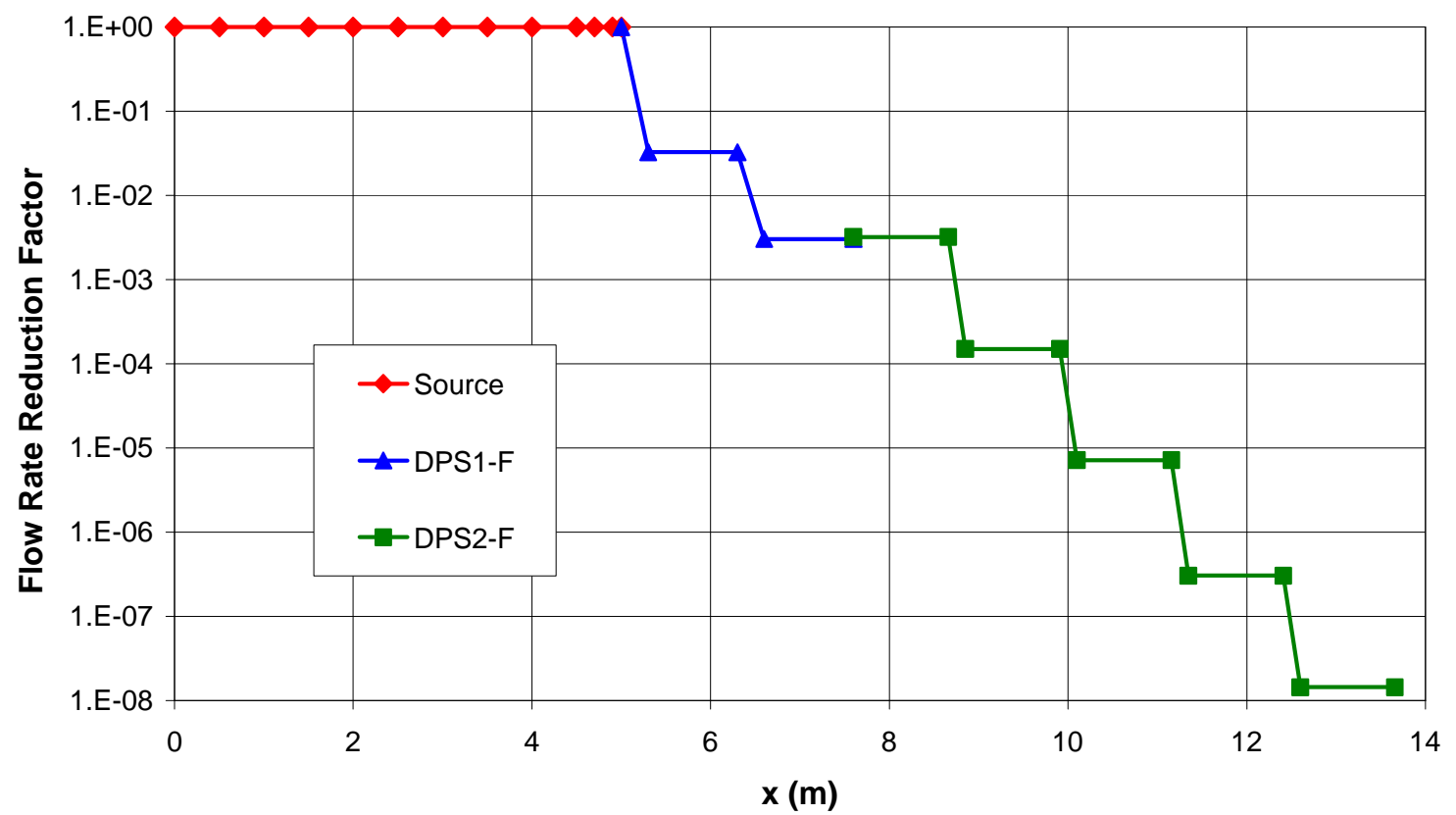

Figure 11. The flow rate reduction factor for the complete differential pumping system. 


\section{REFERENCES}

[1] Osipowicz A, et al., KATRIN: A next generation tritium beta decay experiment with sub-eV sensitivity for the electron neutrino mass. http://arxiv.org/archieve/hep-ex: 0109033, 2001.

[2] KATRIN Collaboration, KATRIN design report 2004, Forschungszentrum Karlsruhe Scientific Report \# FZKA 7090. 2005, http://www-ik.fzk.de/katrin

[3] Gehring R, Bonn J, Bornschein B, et al., The KATRIN magnet system, IEEE Trans. Appl. Superconductivity, 14, 589(2004).

[4] F. Sharipov and V. Seleznev, Rarefied flow through a long tube at any pressure ratio. J. Vac. Sci. Technol. A 12(5), 2933-2935 (1994).

[5] F. Sharipov, Rarefied flow through a long tube at arbitrary pressure and temperature drops. $J$. Vac. Sci. Technol. A 15(4), 2434-2436 (1997).

[6] F. Sharipov, Application of the Cercignani-Lampis scattering kernel to calculations of rarefied gas flows. III. Poiseuille flow and thermal creep through a long tube. Eur.J. Mech. B/Fluids 22, 145-154 (2003).

[7] M. J. Assael, S. Mixafendi, and W. A. Wakeham, "The Viscosity and thermal conductivity of Normal hydrogen in the Limit of Zero Density," J. Phys. Chem. Ref. Data 15, 1315-1322 (1986).

[8] M. J. Assael, S. Mixafendi, and W. A. Wakeham, "The Viscosity of Normal Deuterium in the Limit of Zero Density,” J. Phys. Chem. Ref. Data 16, 189-192 (1987).

[9] J. M. J. Coremans, A. van Itterbeek, J. J. M. Beenakker, H. F. P. Knaap, and P.Zandbergen, “The viscosity of gaseous $\mathrm{He}, \mathrm{Ne}, \mathrm{H}_{2}$ and $\mathrm{D}_{2}$ below $80 \mathrm{~K}$," Physica 24, 557-576 (1958).

[10] X. Luo, C. Day, V. Hauer, O.B. Malyshev, R.J. Reid, F. Sharipov. Monte Carlo simulation of gas flow through the KATRIN DPS2-F differential pumping system. Vacuum 80 (2006) pp. 864-869.

[11] MOLFLOW, supplied by R. Kersevan, ESRF, France, private communication.

[12] MOVAK3D, ver. 6.04/2004, G. Class, Germany, private communication. 